\title{
Chemokines: the times they are a-changin'
}

\author{
Gabriel Márquez and Carlos Martínez-A.
}

\begin{tabular}{|c|}
\hline Commentary \\
\hline $\begin{array}{c}\text { See related article, Volume 107, } \\
\text { number 5, pages 595-601. }\end{array}$ \\
\hline
\end{tabular}

Department of Immunology and Oncology, Centro Nacional de Biotecnología,

Consejo Superior de Investigaciones Científicas, Campus de Cantoblanco, Madrid, Spain

Address correspondence to: Carlos Martínez-A., Department of Immunology and Oncology, Centro Nacional de Biotecnología,

Consejo Superior de Investigaciones Científicas, Campus de Cantoblanco, Madrid E-28049, Spain.

Phone: 3491-585-4559; Fax: 3491-372-0493; E-mail: cmartineza@cnb.uam.es.

What does a name mean? Don Quixote's reply to Sancho Panza was that a name is whatever you understand it to mean. This response certainly applies to the evolution of our thinking about chemokines over the ten years of their history. Initially perceived as simple chemoattractants, chemokines are now implicated at diverse phases of the immune response, and their significance is becoming apparent in many areas of biomedicine, including cancer, HIV-1 infection, asthma, and cardiovascular disease.

\section{Control of T-cell differentiation and trafficking}

Mobilization of leukocytes from peripheral blood into tissues is an essential requirement for immunity in higher organisms. Extravasation of these cells appears to be a highly regulated, multistep process involving coordinated interactions between leukocytes, endothelial cells, and chemoattractant molecules (1, $2)$. In the case of T cells, activation is initiated by recognizing antigens on the surface of antigen-presenting cells (APCs). Simultaneous costimulatory signals from the APCs, such as those provided by the interactions between the receptor protein CD28 and its ligands CD80 and CD86, are required for the $\mathrm{T}$ cell to proliferate and become functionally specialized. The cytokines in the environment in which T-cell activation takes place influence the outcome of the process. Thus, IL-12 skews CD4 T-cell differentiation toward a Th1 cell phenotype, in which the cells produce IFN- $\gamma$ and act mainly in cell-mediated immune responses. On the other hand, IL-4 leads CD4 T cells toward the Th2 subset; these differentiated cells produce IL-4, IL-5, IL-10, and IL-13, and are involved in humoral immunity, including allergic reactions. The Th cell subsets are functionally different, and the Th1/Th2 cell balance determines the outcome of immune responses directed either at infected tis- sue or, in the case of autoimmune disease, at otherwise healthy cells. As with CD4 cells, CD8 T cells can differentiate to Tc1 or Tc2 cell subsets. Although cytokine production by Tc cell subtypes corresponds to that of the Th subtypes, Tc1 and Tc2 cells have cytotoxic activity.

The Th1/Th2 concept has been instrumental in understanding how the immune system works, and some of the factors that influence Th1 or Th2 polarization have been identified. These include the dendritic cells (DCs), a class of APCs specialized in initiating T-cell activation (3). In both humans and mice, some DC subtypes have been shown to promote distinct Th responses. Human DC1 and mouse CD8 $\alpha^{+}$ DCs induce Th1 polarization, which has been linked to their IL-12-producing ability, whereas human DC2 and mouse CD $8 \alpha^{-}$DCs induce Th2 differentiation $(4,5)$. The costimulatory signal provided by the DCs also participates in the Th1/Th2 polarization, as recently shown for the inducible costimulatory molecule, which binds B7RP and favors Th2 differentiation (6-8). Some of the STAT family proteins are known to be necessary signaling effectors for Th1/Th2 differentiation. Induction of STAT4 by IL-12 is required for developing fully functional Th1 cells $(9,10)$, whereas STAT6, scription factors such as T-bet (T-box expressed in T cells) (14) and GATA-3 (15) are reported to participate in Th1 and $\mathrm{Th} 2$ cell differentiation, respectively. Chemokines probably participate in $\mathrm{T}$-cell polarization, in view of their central role in control of DC trafficking and the fact that IL-4 and IL-12 induce production of distinct chemokines (see below). In addition, studies with CCL2-deficient mice show that this chemokine is critical for development of a Th2 response (16).

T-cell polarization activates expression of the cytokine genes that characterize each Th cell subset, and modulates expression of certain others; not surprisingly, these include chemokine receptor genes. In vitro T-cell differentiation under conditions that favor Th1 or Th2 polarization induces different sets of chemokine receptors, with CXCR3 and CCR5 preferentially expressed in Th1 cells, and CCR3, CCR4, and CCR8 expressed in the Th2 cells (17). For this reason, chemokine receptors have served as useful markers in Th1/Th2 cell studies.

The preferential expression of distinct chemokine receptor complements implies that cells have different abilities to target specific environments in response to chemoattractant cues. In a recent issue of the $J C I$, Kim et al. (18)

\section{Chemokines are now implicated at diverse phases of the immune response, and their significance is becoming apparent in many areas of biomedicine.}

induced by IL-4, is similarly needed for Th2 cell differentiation (11-13). Indeed, the unbalanced $\mathrm{T}$-cell responses of STAT6- and STAT4-deficient mice mimic those of IL-4- and IL-12-deficient mice, respectively. Other tran- show that Bonzo/CXCR6, the newly described receptor for the chemokine CXCL16 (19), is a differential marker for the Th1 and Tc1 cell subsets in vitro and in vivo. In vitro-polarized Bonzo ${ }^{+}$ CD4 $\mathrm{T}$ or CD8 cells typically produce 
IFN- $\gamma$, and few of these cells express CCR7, a chemokine receptor involved in homing to and migration within lymphoid tissues. Hence, Bonzo ${ }^{+}$T cells would be expected to migrate preferentially to nonlymphoid destinations, and indeed, arthritic synovial fluid and inflamed liver are enriched for Bonzo ${ }^{+}$ $\mathrm{T}$ cells. As pointed out by Kim et al. (18), T cells in inflamed synovial fluid are efficient Th 1 cytokine producers, so these new data suggest a role for Bonzo in T-cell homing to chronically inflamed tissues. Consistent with the preferential expression of Bonzo in Th1 cells, the authors also found that DCs prime naive $\mathrm{T}$ cells to express Bonzo and that Bonzo expression can be increased by IL-12 and decreased by IL4. Moreover, Bonzo's ligand, CXCL16, is expressed by DCs (19), suggesting the existence of a feedback mechanism to amplify Th1 cell responses.

\section{Integrating chemokine and cytokine signals}

A number of studies have shown that the ability of DCs to migrate to inflamed areas and then to the draining lymph nodes can be explained in terms of a switch in the chemokine receptors they express (17). Immature DCs mainly express receptors for inflammatory chemokines such as CCR1, CCR2, CCR5, and CCR6, which are then downregulated as the DCs mature. Mature DCs upregulate expression of lymphoid-homing chemokine receptors such as CCR7. Naive T cells also express CCR7, guiding them to lymph nodes, where the CCR7 ligands CCL21 and CCL19 are expressed. Once they reach the lymph nodes, these cells require contact with DCs in order to be primed (20). DCs produce chemokines such as CCL18, which binds a yet unknown receptor in naive $T$ cells, as well as CCL17 and CCL22, which bind CCR4, a chemokine receptor expressed by a memory T-cell subset. The importance of coordinated cell movements to the outcome of the immune response was clearly illustrated in a recent study on CCR7 expression in spleen Th1 and Th2 cells (21). Naive and Th1 cells express CCR7 and home to the periar- teriolar lymphoid sheath, whereas Th2 cells, which do not express CCR7, home to the periphery of T-cell zones, in the vicinity of B-cell follicles. Forced expression of CCR7 in Th 2 cells results in a Th1-like splenic localization pattern and yields Th2 cells that are impaired in their ability to help B cells in vivo.

Growing evidence points toward the existence of an intricate network connecting chemokine and cytokine signaling in the regulation of the immune response (22). Cytokines have been shown to promote chemokine expression; for instance, IFN- $\gamma$ induces production of CXCL9 and CXCL10, which attract Th1 cells through their interaction with CXCR3. Similarly, the Th2 cytokines IL-4 and IL-13 stimulate CCL11 production, resulting in chemoattraction of CCR3-expressing Th2 cells. Chemokines such as CCL4 and CCL5, which bind CCR5, induce IL-12 production in the CD $8 \alpha^{+} \mathrm{DC}$ subset (23), thus influencing their subsequent immunological function. Future studies will uncover new transcription factors that participate in Th1 and Th2 differentiation, as well as details of the genetic programs they activate, and they will provide a more complete picture of the molecular elements participating in $\mathrm{T}$-cell polarization.

The current identification of a Bonzo $^{+}$subpopulation will allow studies of a T-cell subtype apparently specialized in homing to highly inflamed tissues. Other T-cell subtypes with novel differentiation pathways and specialized functions remain to be discovered, and new roles for chemokines in these cells will undoubtedly come to light as well. Only a decade has passed since the discovery of the first chemokine, but the variety and importance of these molecules in many physiological and pathological situations are being progressively unraveled. This opens up the possibility of designing a new generation of anti-inflammatory drugs based on chemokine activities. It will be exciting to see the clinical applications derived from this knowledge in the next few years and to learn what further surprises the chemokines and their receptors hold in store for us.
1. Springer, T.A. 1994. Traffic signals for lymphocyte recirculation and leukocyte migration: the multistep paradigm. Cell. 76:301-314.

2. Sánchez-Madrid, F., and del Pozo, M.A. 1999 Leukocyte polarization in cell migration and immune interactions. EMBOJ. 18:501-511.

3. Banchereau, J., and Steinman, R.M. 1998. Dendritic cells and the control of immunity. Nature. 392:245-252.

4. Rissoan, M.C., et al. 1999. Reciprocal control of T helper cell and dendritic cell differentiation. Science. 283:1183-1186.

5. Maldonado-López, R. et al. 1999. CD8 $\alpha^{+}$and $\mathrm{CD} 8 \alpha$ - subclasses of dendritic cells direct the development of distinct $\mathrm{T}$ helper cells in vivo. $J$ Exp. Med. 189:587-592.

6. Dong, C., et al. 2001. ICOS co-stimulatory receptor is essential for T-cell activation and function. Nature. 409:97-101.

7. McAdam, A.J., et al. 2001. ICOS is critical for CD40-mediated antibody class switching. Nature. 409:102-105.

8. Tafuri, A., et al. 2001. ICOS is essential for effec tive T-cell responses. Nature. 409:105-109.

9. Thierfelder, W.E., et al. 1996. Requirement for Stat 4 in interleukin-12 mediated responses of natural killers and T cells. Nature. 382:171-174

10. Kaplan, M.H., Sun, Y.L., Hoey, T., and Grusby, M.J. 1996. Impaired IL-12 responses and enhanced development of Th2 cells in Stat4-deficient mice. Nature. 382:174-177.

11. Kaplan, M.H., Schindler, U., Smiley, S.T., and Grusby, M.J. 1996. Stat6 is required for mediating responses to IL-4 and for the development of Th2 cells. Immunity. 4:313-319.

12. Takeda, K., et al. 1996. Essential role of Stat6 in IL4 signaling. Nature. 380:627-630.

13. Shimoda, K., et al. 1996. Lack of IL-4 induced Th2 response and IgE class switching in mice with disrupted Stat6 gene. Nature. 380:630-633.

14. Szabo, S.J., et al. 2000. A novel transcription factor, T-bet, directs Th1 lineage commitment. Cell. 100:655-669.

15.Zheng, W.P., and Flavell, R.A. 1997. The tran scription factor GATA-3 is necessary and sufficient for Th2 cytokine gene expression in CD4 $\mathrm{T}$ cells. Cell. 89:587-596.

16. Gu, L., et al. 2000. Control of Th2 polarization by the chemokine monocyte chemoattractant protein-1. Nature. 404:407-411.

17. Sallusto, F., Mackay, C.R., and Lanzavecchia, A 2000. The role of chemokine receptors in primary, effector, and memory immune responses. Annu. Rev. Immunol. 18:593-620.

18. Kim, C.H., et al. 2001. Bonzo/CXCR6 expression defines type 1-polarized T-cell subsets with extralymphoid tissue homing potential. J. Clin. Invest. 107:595-601.

19. Matloubian, M., David, A., Engel, S., Ryan, J.E., and Cyster, J.G. 2000. A transmembrane CXC chemokine is a ligand for HIV-coreceptor Bonzo. Nature Immunology. 1:298-304.

20. Cyster, J.G. 1999. Chemokines and cell migration in secondary lymphoid organs. Science. 286:2098-2102.

21. Randolph, D.A., Huang, G., Carruthers, C.J.L. Bromley, L.E., and Chaplin, D.D. 1999. The role of CCR7 in Th1 and Th2 cell localization and delivery of B cell help in vivo. Science. 286:2159-2162.

22. Mackay, C.R. 2001. Chemokines: immunology's high impact factors. Nature Immunology. 2:95-101.

23. Aliberti, J., et al. 2000. CCR5 provides a signal for microbial induced production of IL- 12 by CD $8 \alpha^{+}$ dendritic cells. Nature Immunology. 1:83-87. 